\title{
Very Late Stent Thrombosis Related to Fracture of a Sirolimus-Eluting Stent
}

\author{
Chang-Wook Nam, MD ${ }^{1}$ Kwon-Bae Kim, $\mathrm{MD}^{1}$ and In-Sung Chung, MD² \\ ${ }^{1}$ Department of Internal and ${ }^{2}$ Industrial Medicine, College of Medicine, Keimyung University, Daegu, Korea
}

\begin{abstract}
Although late stent thrombosis is a very rare complication after a drug-eluting stent implantation, this could induce significant sequelae. Several mechanisms for very late stent thrombosis have been suggested. We experienced a new possible mechanism of very late stent thrombosis after the implantation of a sirolimus-eluting stent. A sirolimus-eluting stent fracture might induce mechanical injury of the endothelium and then thrombotic occlusion at 24 months after the stent procedure. (Korean Circulation J 2007;37:385-387)
\end{abstract}

KEY WORDS : Thrombosis ; Stents ; Sirolimus.

\section{Introduction}

Drug-eluting stents(DESs) are commonly used in daily practice during percutaneous coronary intervention(PCI). Several significant complications after DES implantations have been recently reported. Among them, stent thrombosis(ST) is a rare and serious complication. A stent fracture is also a very rare event after a DES implantation. We report here on a case of very late stent thrombosis that occurred in a patient 24 months after a sirolimus-eluting stent(SES) implantation, and this might have been related to a stent fracture.

\section{Case}

A 58-year old male was admitted with an inferior ST segment elevation myocardial infarction(STEMI). He had been transferred to our hospital due to 4 hours of sustained chest pain. The initial ECG exhibited ST segment elevation in the inferior leads during sinus rhythm. Two years prior to his admission, he had undergone two stent implantations to the right coronary artery(RCA) because of an inferior STEMI.

Two years previously, he was transferred to our hospital for the management of an inferior wall STEMI after thrombolytic therapy had been performed for treating the inferior STEMI. As the cardiovascular risk

Received : May 3, 2007

Revision Received : May 22, 2007

Accepted : June 27, 2007

Correspondence : Kwon-Bae Kim, MD, Department of Industrial Medicine,

College of Medicine, Keimyung University, 194 Dongsan-dong, Jung-gu,

Daegu 700-821, Korea

Tel: 82-53-250-8040, Fax: 82-53-250-7034

E-mail:ncwcv@dsmc.or.kr factors, he had a history of hypertension and hyperlipidemia and he was a current smoker. The coronary angiogram revealed two focal stenotic lesions in the proximal and mid RCA(Fig. 1A). After predilation with a $3.0 \mathrm{~mm}$ balloon, a $3.5 \times 23 \mathrm{~mm}$ SES (Cypher, Cordis, Miami Lakes, Florida, USA) was implanted at $16 \mathrm{~atm}$ in the mid RCA, and a $4.0 \times 18 \mathrm{~mm} \mathrm{BX-}$ Velocity(Cypher, Cordis, Miami Lakes, Florida, USA) was implanted at $16 \mathrm{~atm}$, without overlapping, in the proximal RCA after predilation with a $3.0 \mathrm{~mm}$ balloon. There were no events during the 8 month clinical followup. He underwent a follow-up coronary angiogram 8 months later as part of a workup for noncardiac surgery. There was minimal neointimal hyperplasia around both stents(Fig. 1B). Two months later, his antiplatelet therapy was discontinued for performing a left nephrectomy that was due to multiple renal stones and obstructive uropathy. After the operation, he received aspirin 100 mg daily as antiplatelet therapy. The 14 month clinical follow-up after the operation was uneventful.

Primary PCI was then performed. The angiogram revealed total occlusion of the mid RCA at the previous SES implantation site(Fig. 1C). A floppy wire was easily passed. A large amount of thrombi was noted after predilation with a $2.5 \mathrm{~mm}$ balloon. After repeated $3.5 \mathrm{~mm}$ ballooning, intravascular ultrasound was performed to obtain more information on the occluded site. During the automatic pull-back, a sudden loss of a stent strut was noted. There was a stent fracture in the mid portion of the SES and also minimal neointimal hyperplasia(Fig. 2). Because we had already obtained optimal results and there was minimal proliferation of the neointima, we did not perform any additional stent implantations(Fig. 1D). The patient was discharged with 

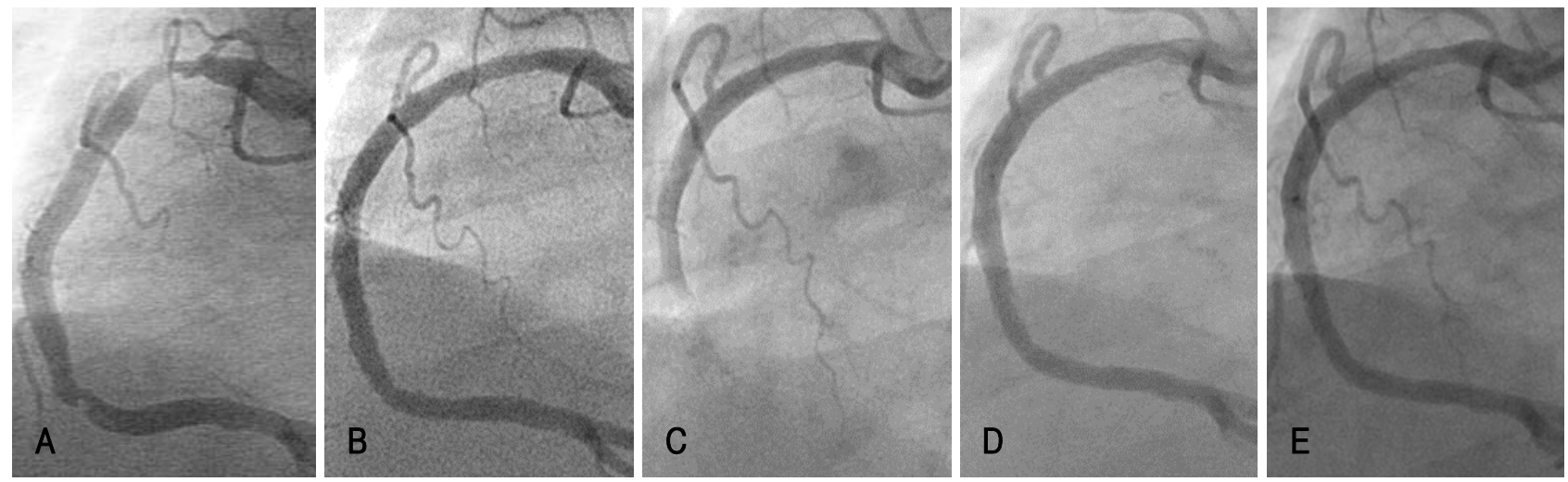

Fig. 1. Angiographic images of the right coronary artery (RCA). A: The initial angiogram showed two focal stenotic lesions at the proximal and mid RCA. B: The follow-up angiogram at 8 months showed a patent stents with minimal luminal narrowing. C: Thrombotic total occlusion in the mid RCA was noted, which was the previous sirolimus-eluting stent implanted site. D: After ballooning, there was minimal residual stenosis. E: The 2nd follow-up angiogram at 6 months showed that the previous ballooning site was patent.

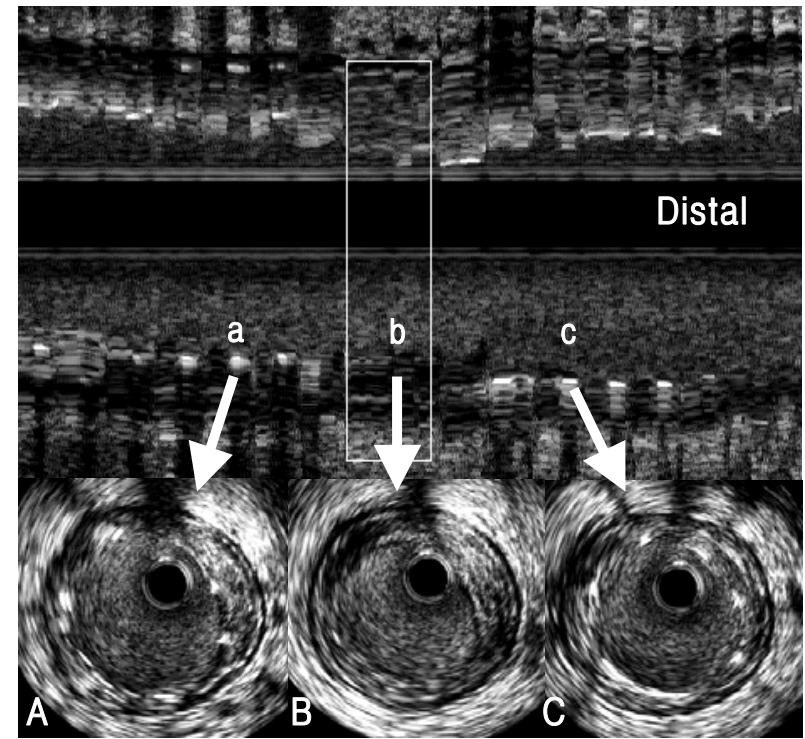

Fig. 2. Top: Longitudinal IVUS reconstruction of the RCA; the white vertical square corresponds to the point of stent fracture. Bottom: A, B, and $\mathrm{C}$ are cross-sectional images corresponding the positions $\mathrm{a}, \mathrm{b}$ and $\mathrm{c}$ on the longitudinal reconstruction. A and C; There are stent struts visible with minimal intimal hyperplasia. $\mathrm{B}$; There is mild concentric plaque with loss of the stent strut, which indicates stent fracture.

taking a triple antiplatelet medication regimen(aspirin $200 \mathrm{mg}$, clopidogrel $75 \mathrm{mg}$, cilostazol $200 \mathrm{mg}$ ). There were no adverse cardiac events noted during the 9 months clinical follow-up. A second follow-up coronary angiography was performed 7 months after the stent thrombosis had occurred; the angiography did not show any evidence of restenosis(Fig. 1E).

\section{Discussion}

The DES has revolutionized the treatment of coronary artery disease, and the randomized studies have show that DES has brought about a significant reduction in the need for repeated revascularizations and a reduced rate of major adverse cardiac event. ${ }^{1)}$ However, concerns have been raised about the safety of those stents in relation to the occurrence of stent thrombosis. ${ }^{2-4)}$ ST with DESs may be associated with significantly higher mortality rates that are related to myocardial infarctions. This rare, but serious complication has drawn the attention of interventional cardiologists. A large portion of STs occur acutely(within 24 hours after the index procedure) or subacutely(between 1 and 30 days after). ${ }^{5}$ However, the focus of concern for the STs was that no end point for late stent thrombosis(LAST: 30 days after) could be observed, even though the incidence of this complication was small. A recent report showed significantly higher rates of death and myocardial infarctions between 6 to 18 months after the procedure in the DES group compared to the BMS group $(4.9 \%$ in the DES group vs. $1.3 \%$ in the BMS group; $p=0.01$ ). ${ }^{4)}$ This astonishing result was interpreted as the clinical consequence of late thrombotic events in the DES implanted patients. ST is mostly related to the discontinuation of the antiplatelet therapy. ${ }^{6}$ However, several different additional factors such as a delayed endothelialization, ${ }^{7)}$ chronic inflammation or hypersensitivity to the polymer, ${ }^{899)}$ late stent malappositioning, ${ }^{710111)}$ and stent underexpansion ${ }^{12)}$ also affect this serious complication. Most of the mechanisms are hypothetical, and especially those that are concerned with very late stent thrombosis, and so more experience and data are needed to understand this phenomenon.

Stent fractures have recently been suggested as a new mechanism of the adverse cardiac events after DES implantation. SES fractures have also been observed by interventional cardiologists. According to the reports, stent fractures might occur with a very tortuous or greatly moved vessels that induce excessive mechanical stress during the contractions and flexion of the heart, 
heavy calcified lesions, long stents, overlapping sites of the stents, larger-sized ballooning sites and especially balloonmg with high pressures. ${ }^{13)}$ Several cases have been reported in which the stent fractures might have been related to the restenosis after the SES implantation. ${ }^{1314)}$ However, the data regarding stent fractures and restenosis has been very limited up to the present. We experienced eight cases with SES stent fractures, and three of these cases experienced adverse cardiac events. Because of the uncertainty of the related mechanism between stent fractures and adverse cardiac events, delicate attention should be paid to the fracture site.

The case we report here suggests the existence of a new potential mechanism for very late stent thrombosis (occurring more than 1 year after the procedure) after the implantation of an SES. Stent fractures might induce mechanical injury to the endothelium, which could produce a stent thrombosis. Because our patient's fracture developed in the late period of the clinical followup, the antiplatelet therapy may have been insufficient to prevent thrombosis formation. Therefore, longer dual antiplatelet therapy may be needed for the groups of patients with a high risk for stent fractures.

Because no IVUS examination was performed during the first follow-up coronary angiogram, the stent fracture may have existed prior to that event. If so, the two events may have developed simultaneously apart from each other. Another possible mechanism of this event is that chronic inflammation, which developed at the fracture site, might have induced atherosclerotic plaque growth and rupture. However, there was minimal intimal hyperplasia observed on the follow-up angiogram. The possibility of plaque formation at the fracture site after the follow-up angiogram was slight. Even though the fracture had previously existed, thrombotic occlusion developed at the fracture site. The stent fracture may have affected or caused some part of the very late stent thrombosis in this patient. However, further experience is required to understand the definite mechanism.

In conclusion, we would like to suggest a new possible mechanism for very late stent thrombosis associated with a stent fracture. In the era of DESs, several special conditions might exist such as the possible need for a longer course of dual antiplatelet therapy than the usual recommended course for the patient groups with a high risk for stent thromboses or stent fractures. Careful selection of the stent length, type and positioning may also be required for special coronary situations. Further studies and collection of more data should be done to clarify whether this is an important issue or only a sporadic observation.

\section{REFERENCES}

1) Morice MC, Serruys PW, Sousa JE, et al. A randomized comparison of a sirolimus-eluting stent with a standard stent for coronary revascularization. $N$ Engl J Med 2002;346:1773-80.

2) Colombo A, Corbett SJ. Drug-eluting stent thrombosis: increasingly recognized but too frequently over-emphasized. J Am Coll Cardiol 2006;48:203-5.

3) Pfisterer ME. Presentation of the results of the Basel Stent Costeffectiveness Trial-Late thrombotic events (BASKET-LATE). 55th Annual Scientific Sessions of the American College of Cardiology. Atlanta, Gerogia, March 14; 2006.

4) Ong AT, McFadden EP, Regar E, Jaegere PT, van Domburg RT, Serruys PW. Late angiographic stent thrombosis (LAST) events with drug-eluting stents. J Am Coll Cardiol 2005;45:2088-92.

5) Kuchulakanti PK, Chu WW, Torguson R, et al. Correlates and longterm outcomes of angiographically proven stent thrombosis with sirolimus-and paclitaxel-eluting stents. Circulation 2006;113: 1108-13.

6) Park DW, Park SW. Stent thrombosis in the era of the drugeluting stent. Korean Circ J 2005;35:791-4.

7) Joner M, Finn AV, Farb A, et al. Pathology of drug-eluting stents in humans: delayed healing and late thrombotic risk. J Am Coll Cardiol 2006;48:193-202.

8) Azarbal B, Currier JW. Allergic reactions after the implantation of drug-eluting stents: is it the pill or the polymer? J Am Coll Cardiol 2006;47:182-3.

9) Nebeker JR, Virmani R, Bennett CL, et al. Hypersensitivity cases associated with drug-eluting coronary stents: a review of available drug events and reports (RADAR) project. J Am Coll Cardiol 2006;47:175-81.

10) Virmani R, Farb A, Guagliumi G, Kolodgie FD. Drug-eluting stents: caution and concerns for long-term outcome. Coron Artery Dis 2004;15:313-8.

11) Choi BR, Lee CW, Park SW. Late stent thrombosis associated with late stent malapposition after drug-eluting stenting: a case report. Korean Circ J 2006;36:472-5.

12) Fujii K, Carlier SG, Mintz GS, et al. Stent underexpansion and residual reference segment stenosis are related to stent thrombosis after sirolimus-eluting stent implantation: an intravascular ultrasound study. J Am Coll Cardiol 2005;45:995-8.

13) Sianos G, Hofma S, Ligthart JM, et al. Stent fracture and restenosis in the drug-eluting stent era. Catheter Cardiovasc Interv 2004;61:111-6.

14) Wilczynska J, Rdzanek A, Kochman J, Horszczaruk GJ, Pietrasik A, Opolski G. Sirolimus eluting stent fracture following angioplasty of diffuse in-stent restenosis in the right coronary artery. Int J Cardiol 2007;118:126-7. 